\title{
Temperature Dependence of Crystalline Structure and DC Performance in LLDPE/HDPE Blending Material
}

\author{
Kai Zhang, Lisheng Zhong, Jinghui Gao, Lunzhi Li, Liang Cao and George Chen \\ State Key Laboratory of Electrical Insulation and Power Equipment \\ Xi'an Jiaotong University \\ Xi'an, Shaanxi 710049, China
}

\begin{abstract}
Thermoplastic blending material is considered as a recyclable environmental-friendly potential candidate for future direct current (DC) cable insulation. Although such a noncrosslinking material has been proved to be superior in certain properties compared with cross-linked polyethylene (XLPE), one of the central concerns for the application of this material system lies in its DC performance at high temperature. In this paper, we investigate the DC conductivity and breakdown strength of a thermoplastic linear lowdensity polyethylene (LLDPE)/high-density polyethylene (HDPE) blending material under temperature range from 30 to $90^{\circ} \mathrm{C}$, the results show that this blend exhibits better temperature-stability in DC performance, and it shows lower conductivity and higher breakdown strength compared with XLPE. The crystalline structure is studied through $X$-ray diffraction (XRD) and differential scanning calorimetry (DSC). The results show that the LLDPE/HDPE blend exhibits a co-crystalline structure with higher crystallinity and larger microcrystal, and when temperature rising, crystallinity and microcrystal size of blend decreases slower compared with XLPE. Such results might enable further application of the LLDPE/HDPE blend on DC cable insulation.
\end{abstract}

Index Terms - polyethylene, temperature dependence, breakdown strength, conductivity, co-crystalline, crystalline structure

\section{INTRODUCTION}

HIGH voltage direct current (HVDC) transmission system, owing to its advantages in delivering electric energy with low loss over long distance and high integrating of renewable energy, has been becoming a fast-growing technology in modern electric industry [1-3]. As one of the key equipment for power grid, HVDC cable has been triggering tremendous research interests nowadays. The cable insulation mainly relies on XLPE material, which exhibits high breakdown strength, low dielectric loss and favorable mechanical properties [4]. Nevertheless, because of the crosslinking process, manufacture of extruded XLPE cable consumes high energy and the byproducts residue in XLPE insulation as impurities [5], which need a long time degassing process to be removed before cable installation. Such limitations may limit XLPE in extra high voltage DC cable insulation application.

Thermoplastic polymer blend material exhibits favorable advantages as potential eco-friendly cable insulation material due to low energy consumption in manufacturing and reduction in greenhouse gas emission during the whole life cycle [6]. Hence, intensive investigations have been performed to evaluate its performance aiming to find its application as cable insulation

Manuscript received on 30 June 2018, in final form 23 August 2018, accepted 23 August 2018. Corresponding author: L. Zhong. material. Hosier et al and Dodd e. al have found isothermal crystallization of a polyethylene blend can enhance its $\mathrm{AC}$ ramp breakdown strength and retarded electrical tree growth $[7,8]$. Li et al have found that a $70 \%$ LLDPE $/ 30 \%$ HDPE blend shows an optimal mechanical and AC electrical properties which is superior to XLPE $[9,10]$. Further study on electrical and water treeing tests have shown that such blend has better anti-aging performance compared to XLPE [11].

Recently, thermoplastic polyethylene materials have been found potential application as cable insulation material for HVDC cables. Zhang et al have pointed out that certain LLDPE/HDPE blend shows higher DC breakdown strength than certain XLPE in thin film tests [12]. Vaughan et al have studied DC performance of polyethylene blend and associated mini-cables, which show superior DC breakdown strength compared to that in XLPE [13, 14]. It should be noticed that although recent studies show the potential application for thermoplastic polyethylene blends as HVDC cable insulation materials, there is still lack of investigations on temperature characteristic of DC performance, which is one of crucial factors for the HVDC cable design at elevated temperatures during operation.

In this paper, the temperature dependence of $\mathrm{DC}$ conductivity and breakdown strength of a LLDPE/HDPE blend are investigated. Moreover, the crystalline structure study reveals the mechanism for the variation on DC performance with temperature. 


\section{EXPERIMENTAL METHOD}

\subsection{SAMPLE PREPARATION}

The LLDPE/HDPE blend with antioxidant (hereinafter referred to as BLEND) was fabricated using melt-blending method through a twin-screw extruder. The starting materials were $70 \%$ LLDPE (commercial pellet products with density of $0.923 \mathrm{~g} \cdot \mathrm{cm}^{-3}$ and melt index of $0.25 \mathrm{~g} / 10 \mathrm{~min}$ ) and $30 \%$ HDPE (commercial pellet products with density of $0.945 \mathrm{~g} \cdot \mathrm{cm}^{-3}$ and melt index of $0.75 \mathrm{~g} / 10$ min) in mass ratio. The produced pellets, neat LLDPE and HDPE pellets were then press-moulded into thin film specimen at $170^{\circ} \mathrm{C} / 10 \mathrm{MPa}$ and cooled to room temperature in air. The XLPE specimen was peeled from the insulation layer of a $160 \mathrm{kV} \mathrm{HVDC}$ cable. BLEND and XLPE specimens were used in the following tests. Before tests, all samples thermally pretreated at $70^{\circ} \mathrm{C}$ for 24 hours.

\subsection{CHARACTERIZATION}

DSC was employed to investigate the melting and crystallization process by using a TA Instruments Q200 differential scanning calorimeter. A series of LLDPE/HDPE blends in different compositions (LLDPE content from $10 \%$ to 90\%) and neat LLDPE, HDPE were studied. The sample mass for test was $5 \mathrm{mg} \pm 0.5 \mathrm{mg}$. DSC test was conducted under nitrogen atmosphere with a temperature range of $20-200{ }^{\circ} \mathrm{C}$ with a heating rate of $10^{\circ} \mathrm{C} / \mathrm{min}$.

XRD was employed to analyze the crystalline structure of material at different temperature conditions $\left(30,50,70,90^{\circ} \mathrm{C}\right)$ by using a Bruker D8 X-ray diffractometer. The scanning range was from 12 to $35^{\circ}$ and the scanning rate was $9.6^{\circ} / \mathrm{min}$.

Dynamic thermo-mechanical (DMA) test was employed by using a Mettler Toledo DMA/SDTA 1+ system. The test sample was $4 \mathrm{~mm}$ in thickness and fixed by a dual cantilever fixture. DMA test was undertaken at a temperature range from 20 to $140^{\circ} \mathrm{C}$ with a heating rate of $2^{\circ} \mathrm{C} / \mathrm{min}$.

The conduction currents at different temperature $(30,50,70$, $90^{\circ} \mathrm{C}$ ) under a $20 \mathrm{kV} / \mathrm{mm}$ DC electric field were measured by a Keithley 6517B electrometer. A three-electrode system was used in the DC conductivity test, the diameter of measuring electrode was $24 \mathrm{~mm}$. The test samples were approximately $200 \mu \mathrm{m}$ in thickness. Conduction currents at each temperature condition were measured 3 times. For each sample, the median of the last 60 current data (sampling interval 1 second) during 60 minutes electric filed applying time was considered as the conduction current result.

Breakdown tests were conducted at $30,50,70,90^{\circ} \mathrm{C}$ respectively. A pair of vertical-aligned cylinder electrodes (with a $25 \mathrm{~mm}$ diameter and a $3 \mathrm{~mm}$ radius of curvature at the electrode edge) were fixed in a frame. The test samples (about $150 \mu \mathrm{m}$ thickness) were placed between electrodes. The whole test setup was immersed in a tank filled with insulating oil and put into an oven. The applied voltage ramp rate was $1.5 \mathrm{kV} / \mathrm{s}$ approximately.

\section{RESULTS AND DISCUSSIONS}

\subsection{THE CO-CRYSTALLINE STRUCTURE IN BLEND}

In order to investigate the crystalline structure in LLDPE/HDPE blends, the melting and crystallization processes have been analyzed by DSC. Despite the variations of LLDPE/HDPE blend compositions, there is only one melting or crystallization peak in each DSC curve, which infers that LLDPE and HDPE have a good compatibility. The melting and crystallization peak temperatures are depicted as functions of LLDPE content in Figures $1 \mathrm{a}$ and $1 \mathrm{~b}$ respectively. The linear fitting results show that $T_{\mathrm{m}}$ and $T_{\mathrm{c}}$ have linear correlations (correlation coefficient $R^{2}$ are 0.944 and 0.962 respectively) with LLDPE contents in blend compositions. According to the studies on polymer blending behavior $[15,16]$, if melting and crystallization peak temperatures are in linear correlation with blend compositions, it can be considered as a criterion for formation of the co-crystalline structure in binary polymer blend. Therefore, a co-crystalline structure is formed after blending in blend.

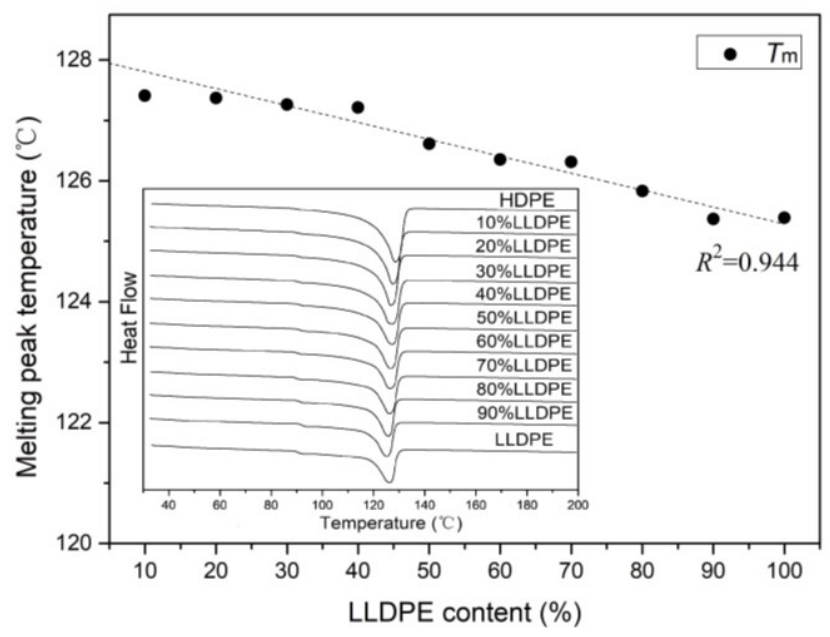

(a) Melting process

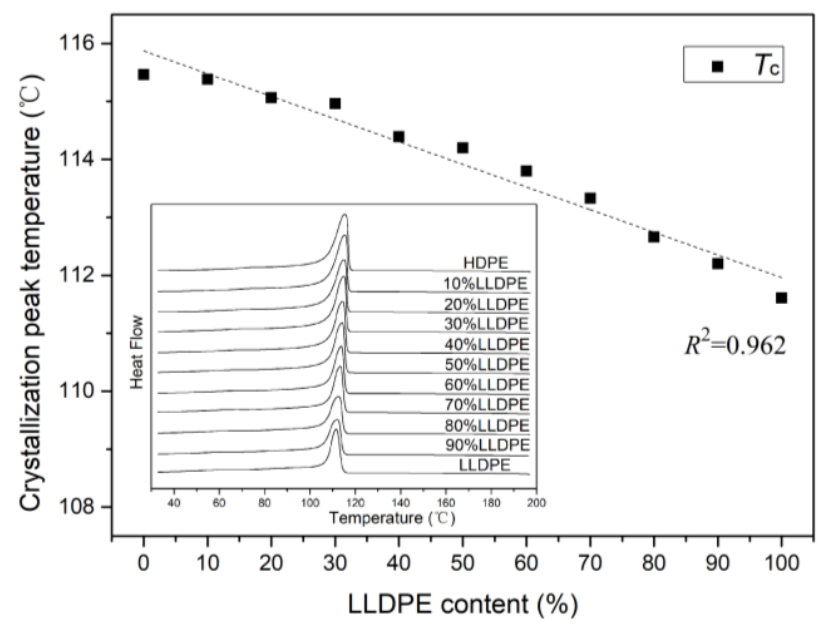

(b) Crystallization process

Figure 1. The variations of melting $\left(T_{\mathrm{m}}\right)$ and crystallization $\left(T_{\mathrm{c}}\right)$ peak temperature with LLDPE/HDPE blend compositions, DSC curves of melting and crystallization process are also illustrated.

\subsection{CRYSTALLINE STRUCTURE CHARACTERISTICS}

In this section, crystalline structure characteristics under temperature range from 30 to $90^{\circ} \mathrm{C}$ are investigated. The normalized XRD patterns are illustrated in Figure 2. 
There are two main diffraction peaks in each XRD pattern. The diffraction peaks near 21 and $24^{\circ}$ correspond to the (110) crystal plane and the (200) crystal plane (hereinafter referred to as (110) and (200) respectively) [17]. The peak near $19^{\circ}$ is caused by the diffuse reflectance of the amorphous region. As temperature increases, the positions of diffraction peaks of both materials shift slightly toward small diffraction angles due to the increasing Bragg spacing (the influence of thermal expansion of sample thickness could be neglected according to calculation result), the diffraction peaks become broader and the diffuse reflectance of the amorphous region is enhanced. It can be found that this XLPE has more obvious diffuse reflectance than that of BLEND. The variations of diffraction peak position and shape reflect the changes of crystalline structure.

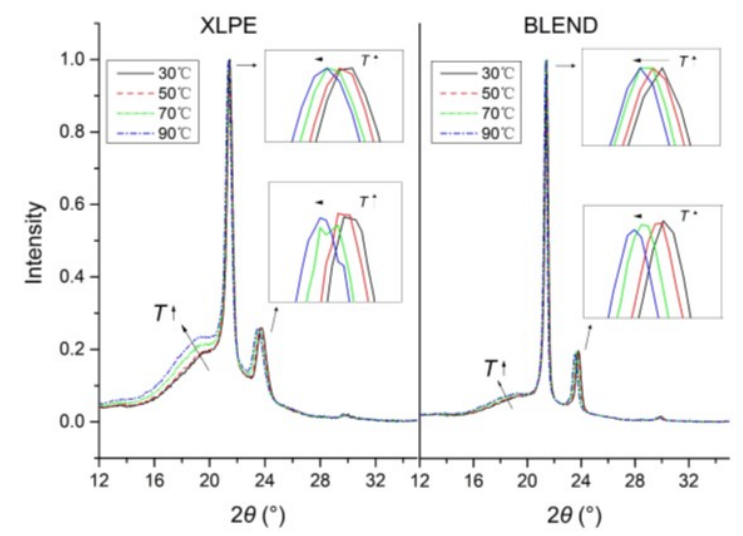

Figure 2. XRD patterns of BLEND and XLPE vary with temperatures.

XRD patterns in Figure 2 are processed by peak fitting, microcrystal size $D$ can be calculated by Scherrer formula [17]:

$D=\frac{K \lambda}{B \cos \theta}$

where the Scherrer constant $K$ is 0.89 and X-ray wavelength $(\mathrm{Cu} \mathrm{K \alpha}$ target) $\lambda$ is $0.15418 \mathrm{~nm} . B$ is half-width of diffraction peak and $2 \theta$ is the angle of diffraction peak.

The crystallinity $X_{\mathrm{c}}$ can be calculated by

$$
X_{\mathrm{c}}=1-\frac{S_{a}}{S_{\mathrm{a}}+S_{\mathrm{c}}} \times 100 \%
$$

where $S_{\mathrm{c}}$ is the sum of crystallization peaks area and $S_{\mathrm{a}}$ is the amorphous peak area.

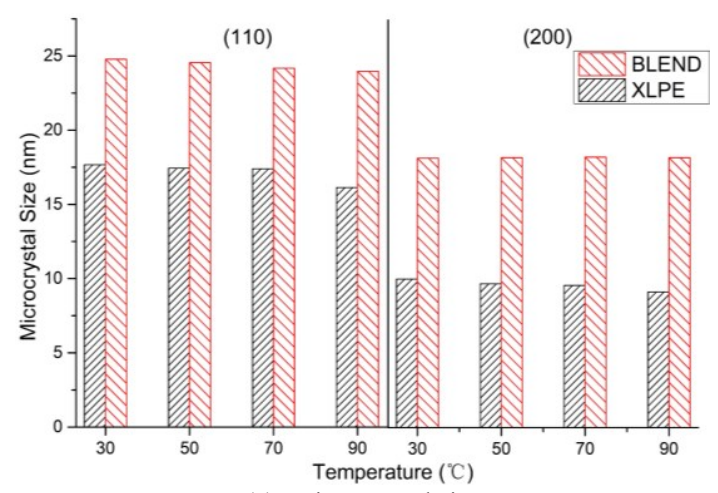

(a) Microcrystal size

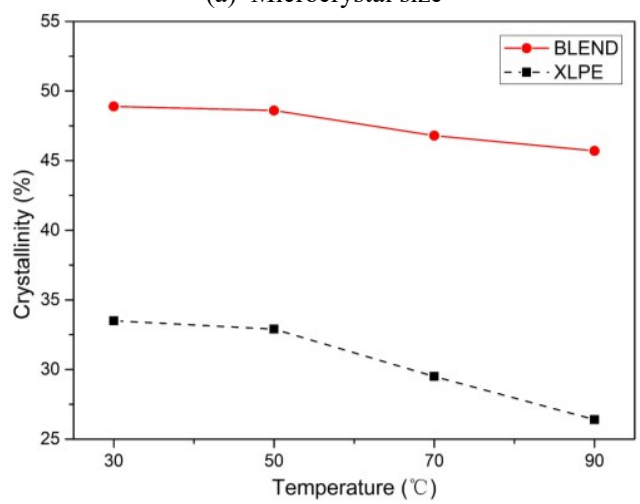

(b) Crystallinity

Figure 3. Microcrystal size and crystallinity vary with temperatures.

Microcrystal size and crystallinity at different temperatures are shown in Figures $3 \mathrm{a}$ and $3 \mathrm{~b}$. According to the calculation results in Table 1, BLEND shows higher crystallinity and larger microcrystal size compared with XLPE, which is different with the general view that the polymer blend would exhibit more pronounced changes in crystallinity with temperature variations than XLPE. The reason may be ascribed to two aspects. First, the matrix LDPE resin used in XLPE has a high degree of branching, which hinders crystallization process. Second, the network structure formed in crosslinking process limits segment motion and arrangement, which does not benefit crystal nucleation and growth. As temperature increases, thermal motions of polymer molecular chains increase gradually and crystalline regions begin to melt, microcrystal size and crystallinity show downward trends. The crystallinity and microcrystal size of BLEND decreases slower than that of $\mathrm{XLPE}$, which shows a higher temperature-stability. It can be

Table 1. Crystalline structure characteristics parameters.

\begin{tabular}{|c|c|c|c|c|c|c|c|c|}
\hline \multirow{2}{*}{ Sample } & \multirow{2}{*}{$T\left({ }^{\circ} \mathrm{C}\right)$} & \multicolumn{2}{|c|}{$2 \theta\left(^{\circ}\right)$} & \multicolumn{2}{|c|}{ Half-width $\left(^{\circ}\right)$} & \multicolumn{2}{|c|}{ Microcrystal size (nm) } & \multirow{2}{*}{ Crystallinity (\%) } \\
\hline & & (110) & $(200)$ & $(110)$ & $(200)$ & (110) & $(200)$ & \\
\hline \multirow{4}{*}{ BLEND } & 30 & 21.440 & 23.808 & 0.323 & 0.442 & 24.773 & 18.179 & 48.9 \\
\hline & 50 & 21.421 & 23.773 & 0.326 & 0.441 & 24.545 & 18.219 & 48.6 \\
\hline & 70 & 21.381 & 23.665 & 0.331 & 0.44 & 24.172 & 18.256 & 46.8 \\
\hline & 90 & 21.363 & 23.603 & 0.334 & 0.441 & 23.954 & 18.213 & 45.7 \\
\hline \multirow{4}{*}{ XLPE } & 30 & 21.464 & 23.752 & 0.453 & 0.801 & 17.665 & 10.030 & 33.5 \\
\hline & 50 & 21.433 & 23.678 & 0.459 & 0.828 & 17.433 & 9.702 & 32.9 \\
\hline & 70 & 21.394 & 23.572 & 0.460 & 0.837 & 17.394 & 9.596 & 29.5 \\
\hline & 90 & 21.368 & 23.499 & 0.496 & 0.877 & 16.131 & 9.157 & 26.4 \\
\hline
\end{tabular}


explained in: (1) Matrix resins of BLEND have less molecular branches and higher regularity, which make the crystal surface energy of BLEND lower than XLPE and leads to a slower crystal melting process. (2) The co-crystalline forms in BLEND enhances the intermolecular interactions, which retards the crystal melting process.

\subsection{TEMPERATURE DEPENDENCE OF DC CONDUCTIVITY}

Conduction currents under temperature range from 30 to $90^{\circ} \mathrm{C}$ are measured and the corresponding conductivities are calculated. The logarithm of conductivities are depicted as functions of the reciprocal of the absolute temperatures and fitted in a linear relationship as shown in Figure 4.

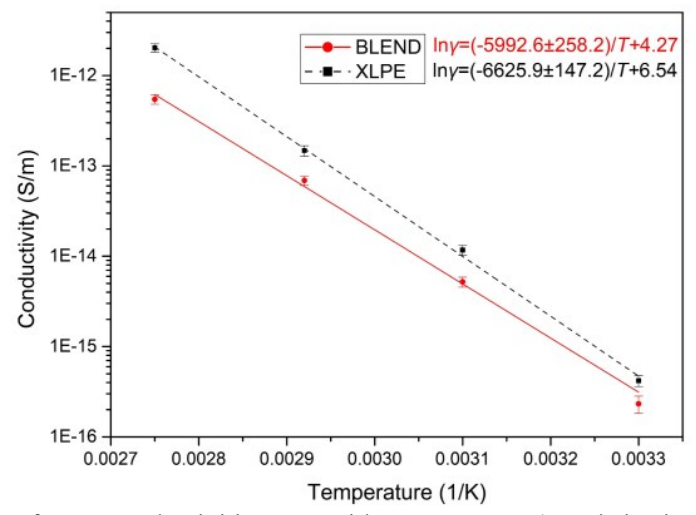

Figure 4. DC Conductivities vary with temperatures ( $\mathrm{X}$ axis is given in reciprocal of the absolute temperature and $\mathrm{Y}$ axis is given in the logarithm of conductivity).

It can be seen that the conductivity of BLEND is lower than that of this XLPE, the conductivities of both materials increase exponentially with temperature rising. The linear relationship between the logarithm of conductivity and the reciprocal of the absolute temperature is in accordance with the Arrhenius-type equation [18]. The activation energy for conductance is calculated. The activation energy of BLEND is $0.517 \pm 0.022 \mathrm{eV}$ which is smaller compared with $0.572 \pm 0.013 \mathrm{eV}$ of XLPE. Therefore, BLEND shows a better temperature-stability in conductivity with temperature variation.

The major reason for differences in DC performance can be contributed to structure variation. Polymer material is composed of crystalline and amorphous regions, which represents a short-range orderly and long-range disorderly structure feature. The energy band only exists in the localized crystalline region, and electron transfers from the conduction band of a localized crystalline region to the conduction band of an adjacent local crystalline region should overcome a degree of potential barrier by means of hopping or tunneling through amorphous region. At relatively low electric field ( $E<100 \mathrm{kV} / \mathrm{mm})$, hopping conduction produced by the thermal excitation electrons plays a dominant role, and the associated conductivity $\gamma$ can be expressed by the following equation [19]:

$\gamma=\frac{n q^{2} d^{2} v}{6 k T} \mathrm{e}^{\frac{-u}{k T}}$

where $k$ is Boltzmann's constant, $n$ is carrier concentration, $q$ is electron charge, $d$ is space between microcrystals, $v$ is thermal vibration frequency of electron and $u$ is energy barrier of microcrystal. According to equation (3), hopping distance have direct influences on conductivity, which is related to crystalline structure. Based on XRD analysis, BLEND exhibits a much more compact structure with larger crystalline region and microcrystal size, which indicates a smaller space between microcrystals hence lower conductivity compared with XLPE. As temperature rising, the crystallinity and microcrystal size of BLEND decreases slower compared with XLPE, therefore BLEND shows a better temperature-stability in conductivity.

\subsection{TEMPERATURE DEPENDENCE OF BREAKDOWN STRENGTH}

Figure 5 shows two-parameter Weibull distributions of breakdown tests vary with temperature ranging from 30 to $90^{\circ} \mathrm{C}$. The scale parameter $E_{0}$ (breakdown strength of $63.2 \%$ cumulative failure probability) and the shape parameter $\beta$ are obtained according to Weibull distributions.

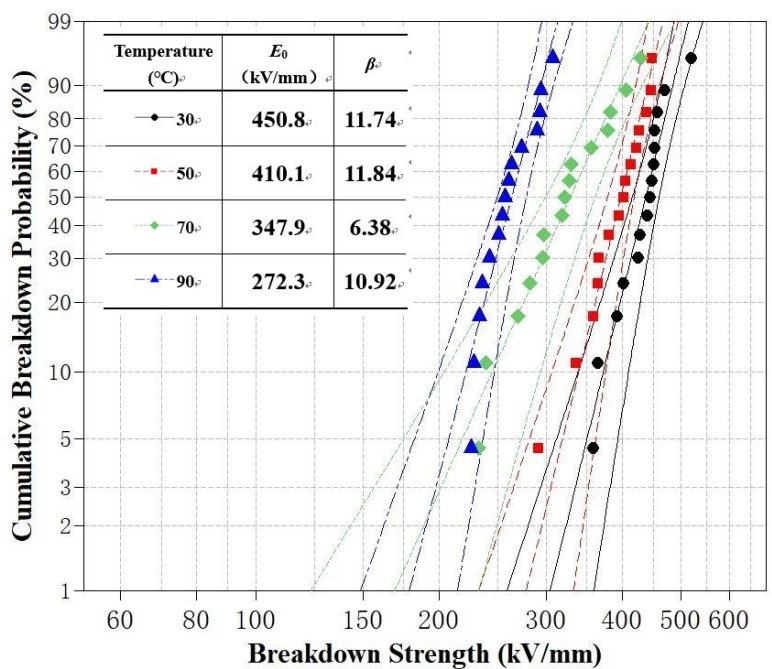

(a) BLEND

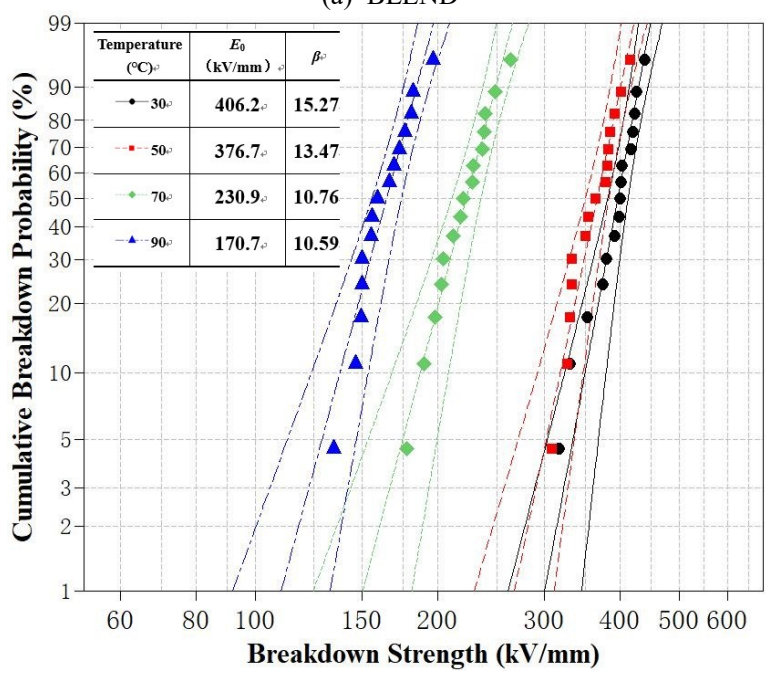

(b) XLPE

Figure 5. Weibull distributions of DC breakdown test under different temperatures ( $95 \%$ confidence interval), the scale parameter $E_{0}$ and the shape parameter $\beta$ are also given.

Figure 6 shows temperature dependence of the breakdown strength. It can be seen that the breakdown strength of BLEND is higher than XLPE across the measured temperature range. 
With the increasing temperature, the breakdown strength decreases in both of the specimens, and the one for BLEND declines slower than that of XLPE. Variation of breakdown strength is discussed in following two aspects which are strongly related to crystalline structure characteristics. On one hand, according to electromechanical breakdown theory, the electromechanical breakdown voltage $V_{\mathrm{em}}$ can be defined as [20]:

$$
V_{e m}=d_{0}\left(\frac{Y}{\varepsilon_{0} \varepsilon_{r} \mathrm{e}}\right)^{\frac{1}{2}}
$$

where $Y$ is the Young's modulus of elasticity and $d_{0}$ is the initial dielectric thickness. In polymer material, there is a positive correlation between crystallinity and Young's modulus. Figure 7 shows the variations of Young's modulus with temperature according to DMA test. BLEND shows higher Young's modulus than XLPE, which is in accordance with crystallinity results at $30 \sim 90^{\circ} \mathrm{C}$. Therefore, it contributes to a higher electromechanical breakdown strength than XLPE. On the other hand, higher crystallinity and larger microcrystal size limit the free volume in BLEND, which leads to the decrease in mean free path and acceleration probability of electron. Breakdown is not easy to be initiated due to electron is limited in accumulating enough energy. As a result, BLEND exhibits higher breakdown strength and better temperature-stability compared with XLPE.

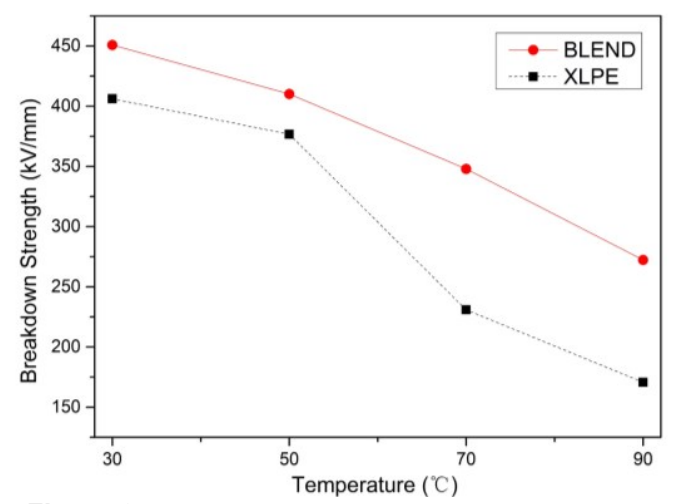

Figure 6. Breakdown strength varies with temperature.

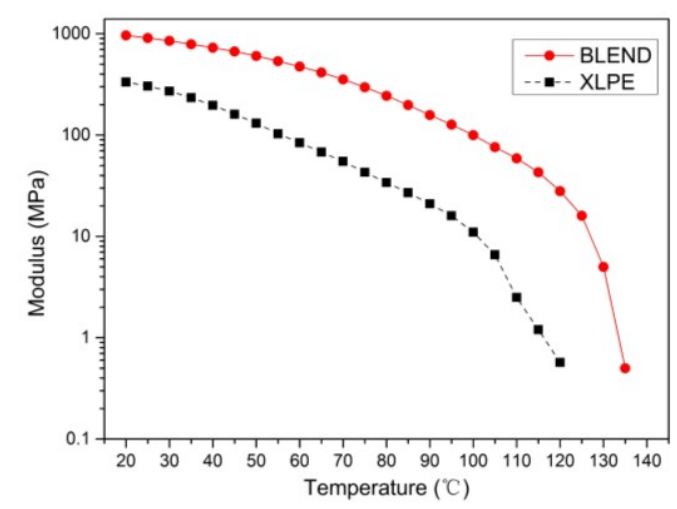

Figure 7. Young's modulus varies with temperature.

\section{CONCLUSIONS}

A 70\% LLDPE/30\% HDPE blend shows better DC performance than XLPE with lower conductivity and higher breakdown strength. Conductivity and breakdown strength of both materials degrade with increasing temperature, while BLEND exhibits a better temperature-stability than XLPE. The reasons for DC performance differences are explained by a cocrystalline structure formed in BLEND, which leads to higher crystallinity and larger microcrystal size compared with XLPE. The co-crystalline structure leads to a shorter average hopping distance, which contributes to a lower conductivity. And it also leads to a higher modulus and smaller free volume, which contributes to higher breakdown strength. Such crystalline structure retards the crystal melting process of BLEND, and guarantees a temperature-stable DC performance.

\section{ACKNOWLEDGMENT}

The authors would like to thank the support of National Key R\&D Program of China (2016YFB0900702).

\section{REFERENCES}

[1] M. P. Bahrman and B. K. Johnson, "The ABCs of HVDC transmission technologies," IEEE Power Energ. Mag., vol. 5, no. 2, pp. 32-44, 2007.

[2] T. J. Hammons, V. F. Lescale, K. Uecker, M. Haeusler, D. Retzmann, K. Staschus and S. Lepy, "State of the Art in Ultrahigh-Voltage Transmission," Proceedings of the IEEE, vol. 100, no. 2, pp. 360-390, 2012.

[3] W. Long and S. Nilsson, "HVDC transmission: yesterday and today," IEEE Power Energ. Mag., vol. 5, no. 2, pp. 22-31, 2007.

[4] H. Orton, "History of underground power cables," IEEE Electr. Insul. Mag., vol. 29 , no. 4 , pp. 52-57, 2013.

[5] C. W. Reed, "An assessment of material selection for high voltage DC extruded polymer cables," IEEE Electr. Insul. Mag., vol. 33, no. 4, pp. $22-$ $26,2017$.

[6] M. Fairhurst, A. Gowadia, G. Stevens, B. Philpot, J. Thomas, J. Pilgrim and P. Lewin, "Integrated development and assessment of new thermoplastic high voltage power cable systems," CIGRE, paper B1-215, 2012

[7] I. L. Hosier, A. S. Vaughan, and S. G. Swingler, "Structure-property relationships in polyethylene blends: the effect of morphology on electrical breakdown strength," Jour. Mater. Sci., vol. 32, no. 17, pp. 4523-4531, 1997.

[8] S. J. Dodd, J. V. Champion, Y. Zhao, A. S. Vaughan, S. J. Sutton, and S.G. Swingler, "Influence of morphology on electrical treeing in polyethylene blends," IEE Proc. Sci. Measur. Tech., vol. 150, no. 2, pp.58-64, 2003

[9] L. Li, K. Zhang, L. Zhong, N. Chen, M. Xu, D. Xie and G. Chen, "Dielectric behaviors of recyclable thermo-plastic polyolefin blends for extruded cables," IEEE $11^{\text {th }}$. Inter. Conf. Prop. Appl. Dielect. Mat.(ICPADM), 2015, pp. 180-183.

[10] K. Zhang, L. Li, L. Zhong, N. Chen, M. Xu, D. Xie, and G. Chen, "The mechanical properties of recyclable cable insulation materials based on thermo-plastic polyolefin blends," IEEE $11^{\text {th }}$. Inter. Conf. Prop. Appl. Dielect. Mat.(ICPADM), 2015, pp 532-535.

[11] L. Li, K. Zhang, L. Zhong, J. Gao, M. Xu, G. Chen, and M. Fu, "Treeing phenomenon of thermoplastic polyethylene blends for recyclable cable insulation materials," AIP Advances., vol. 7, no. 2, pp. 6-9, 2017.

[12] K. Zhang, L. Li, L. Zhong, L. Cao, M. Xu, G. Chen, and M. Fu, "DC dielectric properties of thermo-plastic polyolefin materials," Annu. Rep. Conf. Electr. Insul. Dielectr. Phenom. (CEIDP), 2016, pp 470-473.

[13] C. D. Green, A. S. Vaughan, G. C. Stevens, S. J. Sutton, T. Geussens, and M. J. Fairhurst, "Recyclable power cable comprising a blend of slowcrystallized polyethylenes," IEEE Trans. Dielectr. Electr. Insul., vol. 20, no. 1, pp.1-9, 2013

[14] G. C. Stevens, A. Pye, A. S. Vaughan, C. D. Green, and J. Pilgrim, "High performance thermoplastic cable insulation systems," IEEE Int. Conf. Dielect. (ICD), 2016, pp 272-275.

[15] P. Vadhar, and T. Kyu, "Effects of mixing on morphology, rheology, and mechanical properties of blends of ultra-high molecular weight polyethylene with linear low-density polyethylene," Poly. Engin. Sci. vol. 27, no. 3, pp. 202-210, 1987. 
[16] S. R. Hu, T. Kyu, and R. S. Stein, "Characterization and properties of polyethylene blends I. Linear low-density polyethylene with high-density polyethylene," Journ. Poly. Sci. Part A Poly. Chem. vol. 25, no. 2, pp. 7187, 1987.

[17] Z. Mo, H. Zhang, and J. Zhang, Structure of Crystalline Polymer and Xray Diffraction ( $2^{\text {nd }}$ edition), Science Press, 2003. (in Chinese)

[18] A. D. McNaught, and A. Wilkinson, Compendium of Chemical Terminology ( $2^{\text {nd }}$ edition), Blackwell Scientific Publications, 1997.

[19] W. Jin, Dielectric Physics, Mechanical Industry Press, 1997. (in Chinese)

[20] L. A. Dissado, and J. C. Fothergill, Electrical Degradation and Breakdown in Polymers, Institution of Engineering and Technology, 1992.

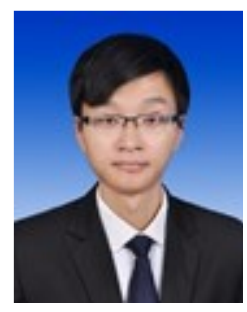

Kai Zhang was born in Shaanxi, China, in 1987. He received the BSc degree and the $\mathrm{MSc}$ degree in electrical engineering from Xi'an Jiaotong University in 2009 and 2012, respectively. He is undertaking the $\mathrm{PhD}$ degree in Xi'an Jiaotong University now. His research interest is on the electrical insulation materials for cable system.

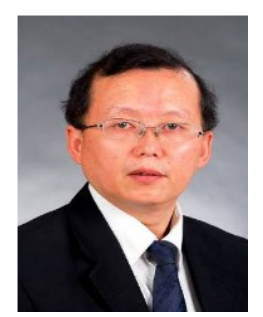

Lisheng Zhong was born in Sichuan, China, in 1961. He received the B. Eng., M. Sc and Ph. D. degrees in electrical engineering, from Xi'an Jiaotong University, respectively in 1983, 1986 and 1997. Currently, he is a professor at the State Key Laboratory of Electrical Insulation and Power Equipment in Xi'an Jiaotong University. His research interests include dielectrics and electrical insulation.

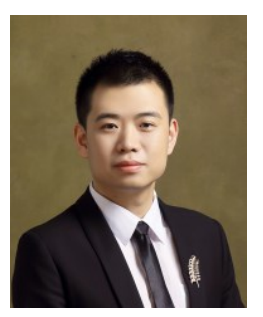

Jinghui Gao was born in Shaanxi, China, in 1984. $\mathrm{He}$ received the B. Eng. and $\mathrm{Ph}$. D. degrees in electrical engineering, from Xi'an Jiaotong University in 2006 and 2012 respectively. Currently, he works as an associated professor at the State Key Laboratory of Electrical Insulation and Power Equipment in Xi'an Jiaotong University. His research interests include functional dielectrics and electrical insulation.

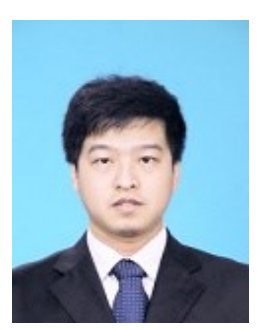

Lunzhi Li was born in Xianyang, China, in 1988. He received the BSc degree of electrical engineering in Xi'an Jiaotong University in 2009. He is now studying his $\mathrm{PhD}$ degree of high voltage and electrical insulation in Xi'an Jiaotong University.

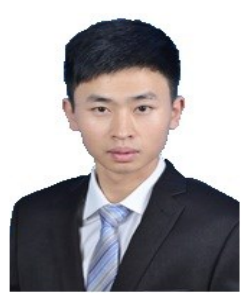

Liang Cao was born in Chengdu, China in 1990. He received the B.E. degree in Electrical Engineering from Xi'an Jiaotong University. Now he is a Ph.D. student of Electrical Engineering of Xi'an Jiaotong University. He has been involved in research on materials for HVDC cable application.

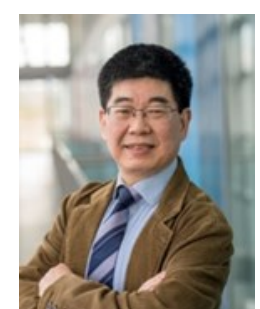

George Chen was born in China in 1961. He received the BEng (1983) and MSc (1986) degrees in electrical engineering from Xi'an Jiaotong University, China. After he obtained the $\mathrm{PhD}$ degree (1990) from the University of Strathclyde, UK, on the work of permanent changes in electrical properties of irradiated lowdensity polyethylene, he joined the University of Southampton as a postdoctoral research fellow and became a senior research fellow subsequently. In 1997 he was appointed as a research lecturer and promoted to a reader in 2002. He is now professor of HV engineering at the University of Southampton and a visiting professor of Xi' an Jiaotong University. Over the years, he has developed a wide range of interests in $\mathrm{HV}$ engineering and electrical properties of materials and has published more than 300 papers. 